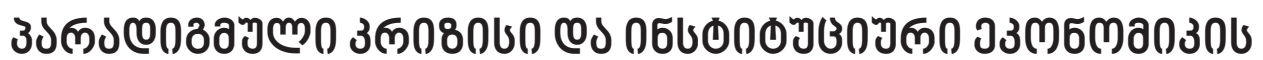

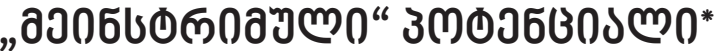

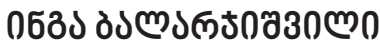

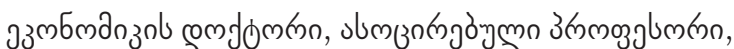

https://doi.org/10.35945/gb.2018.06.015

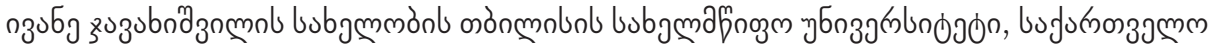

Inga.balarjishvili@tsu.ge

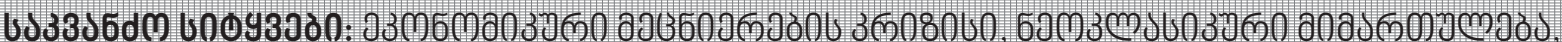

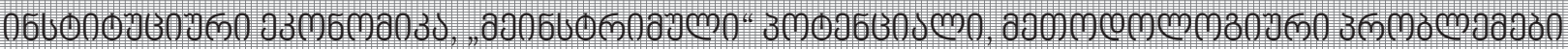

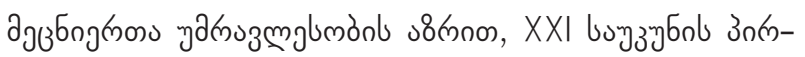

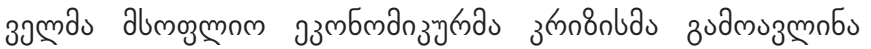

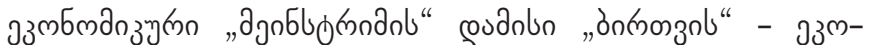

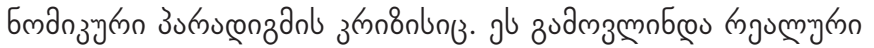

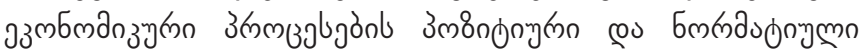

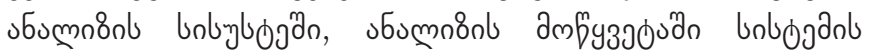

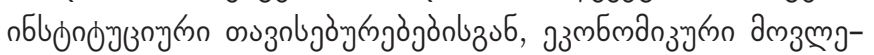

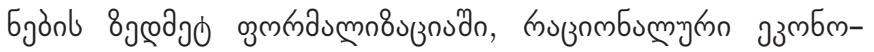

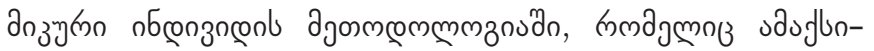

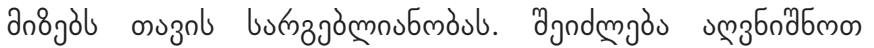

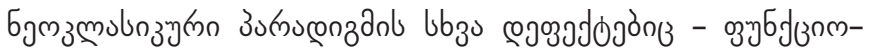

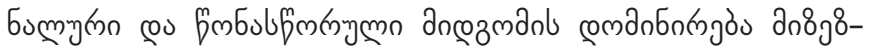

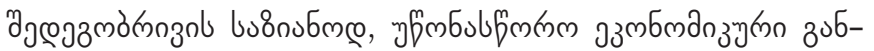

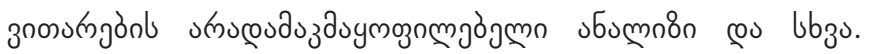

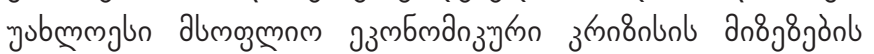

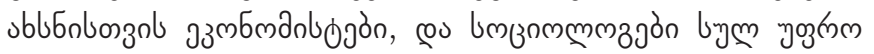

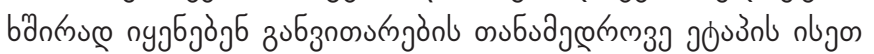

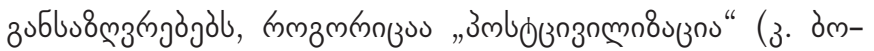

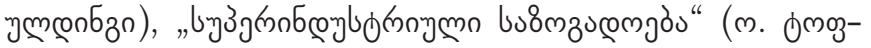

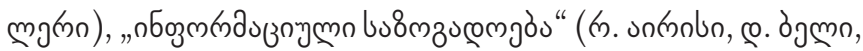

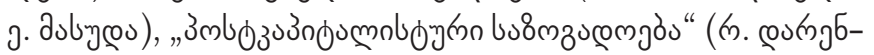

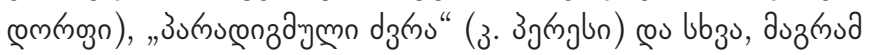

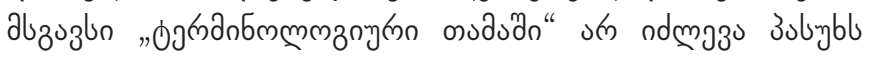

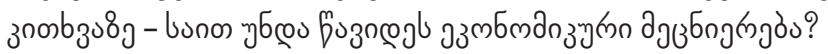

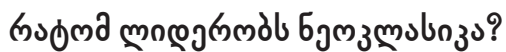

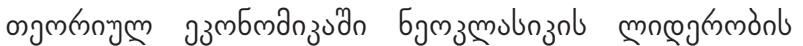

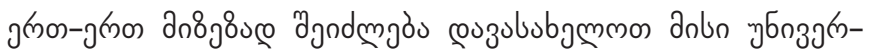

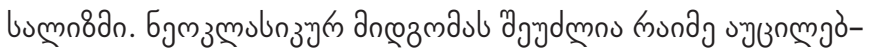

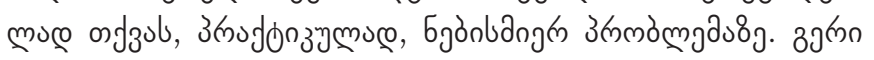

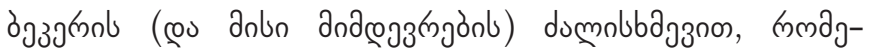

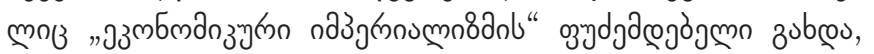

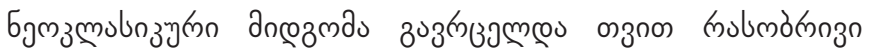

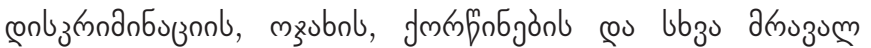

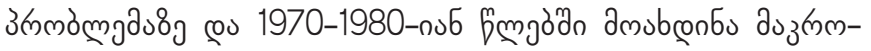

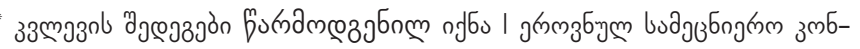

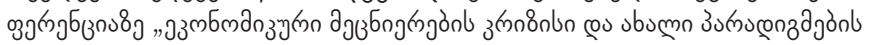
dngos"

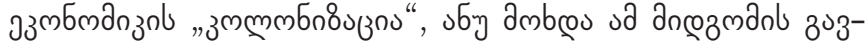

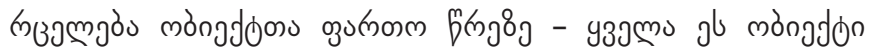

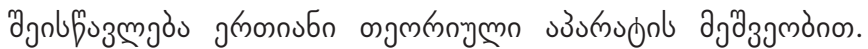

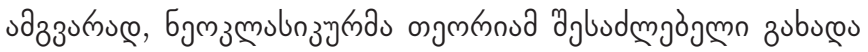

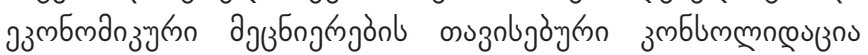

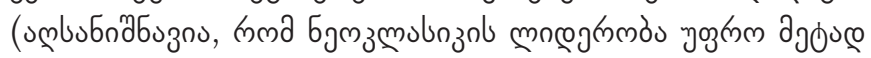
चुbuahб

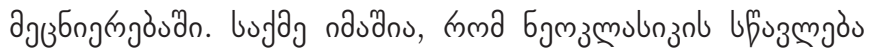

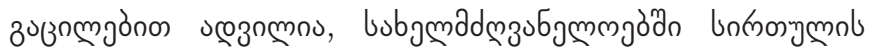

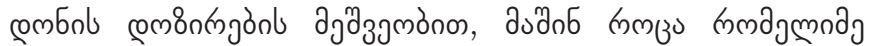

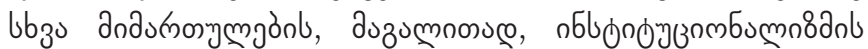

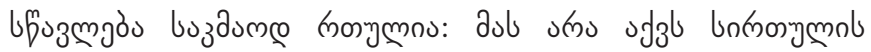

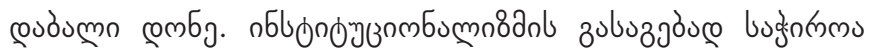

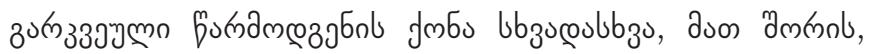

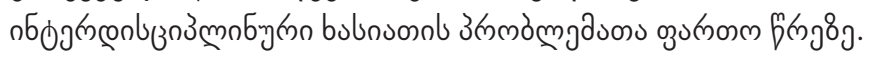

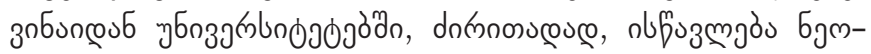

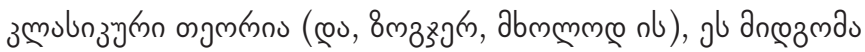

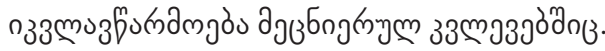

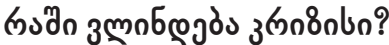

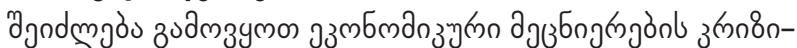
unl zuamz

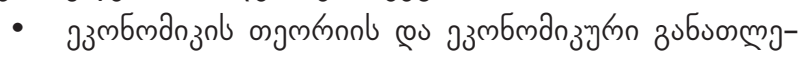

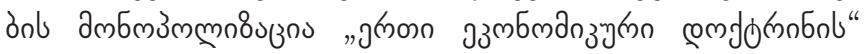

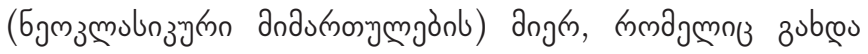

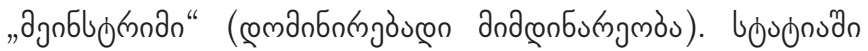

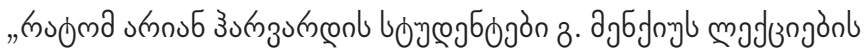

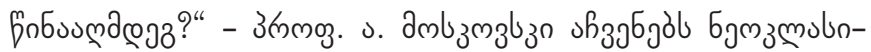

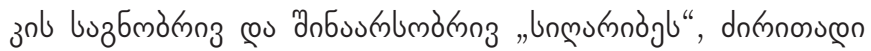

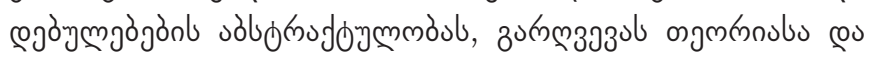

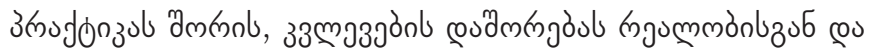

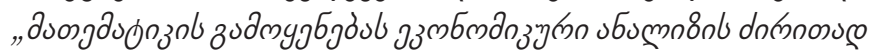

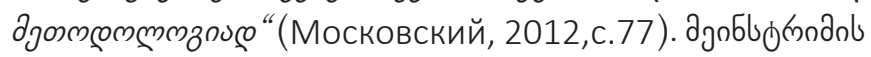

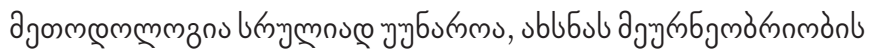

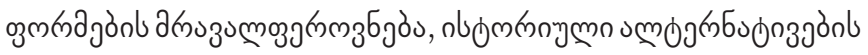
ง ऊl

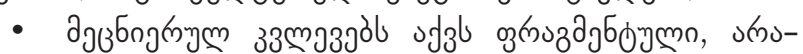

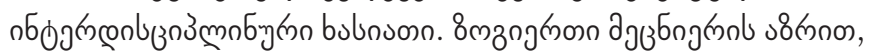




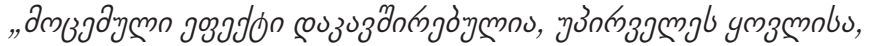

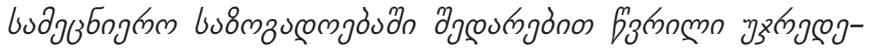

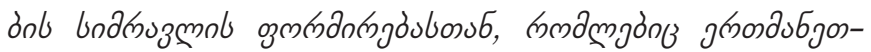

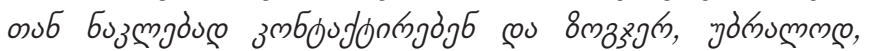

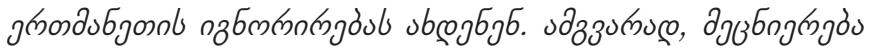

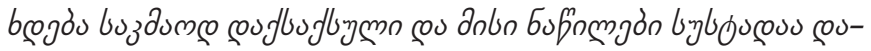

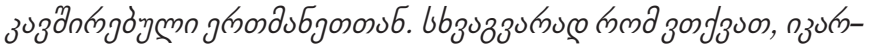

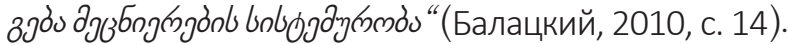

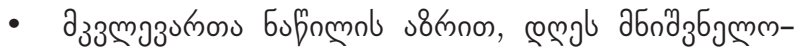

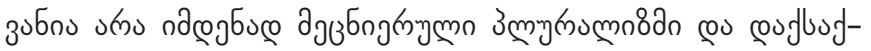

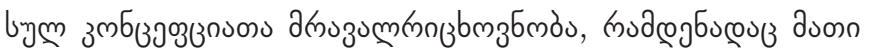

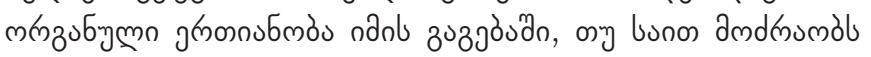

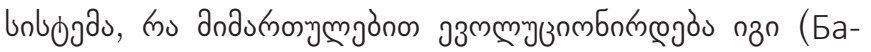

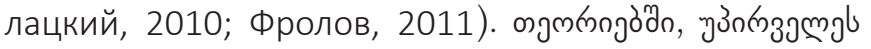

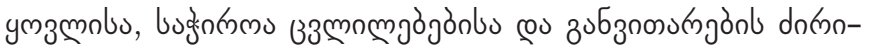

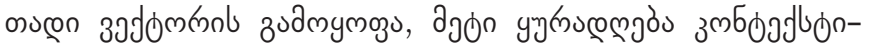

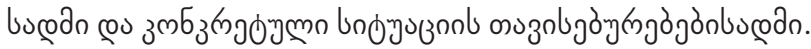

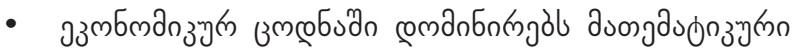

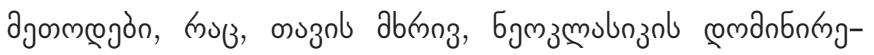

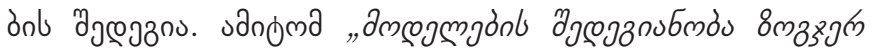

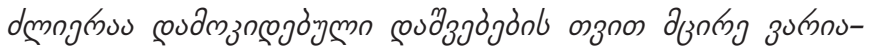

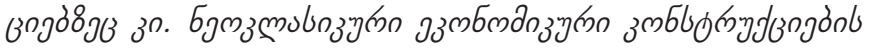

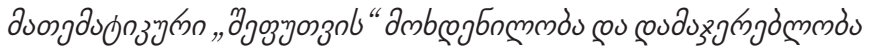

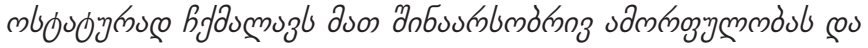

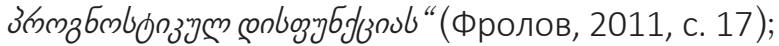

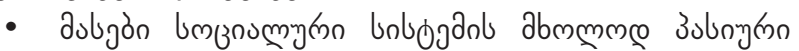

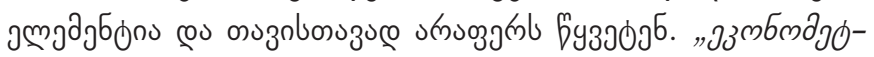

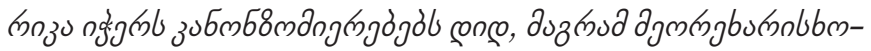

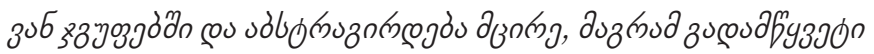

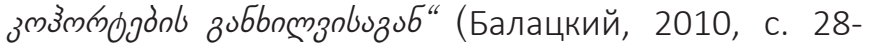

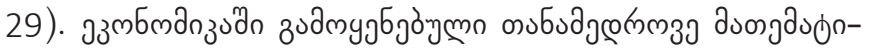

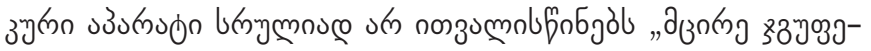

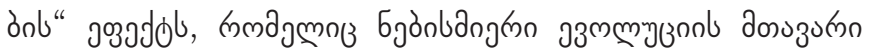
audmdnuzjòjmo dumus.

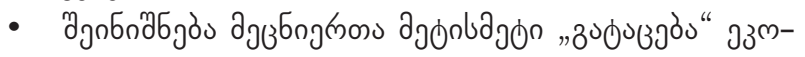

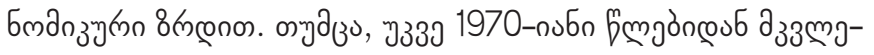

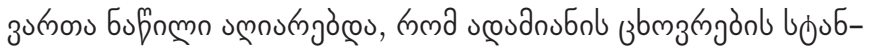

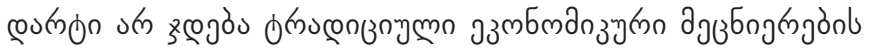

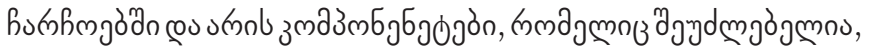

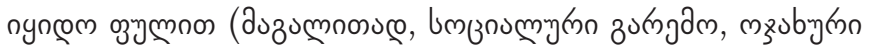

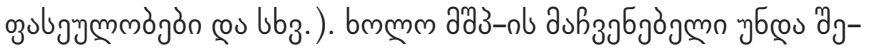

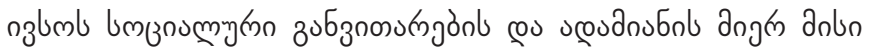

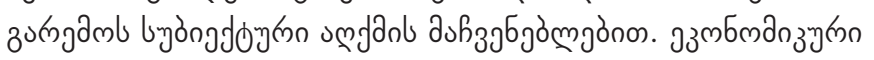

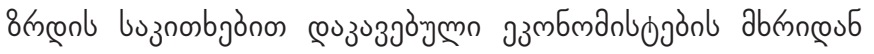

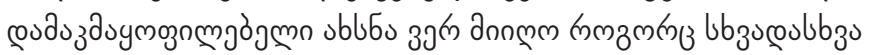

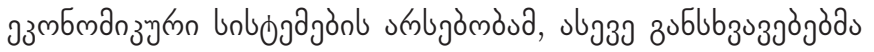

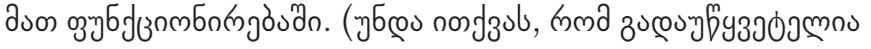

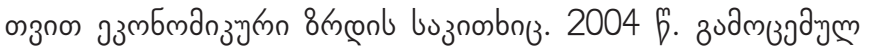

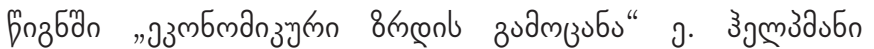

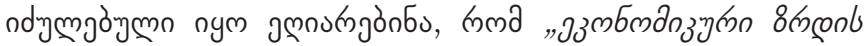

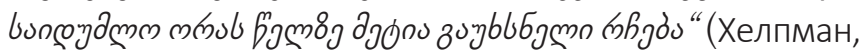

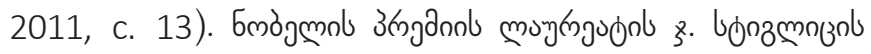

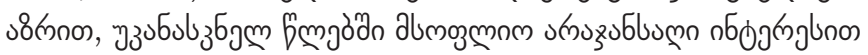

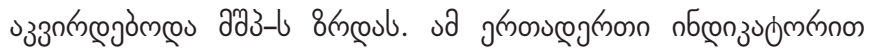

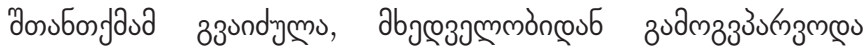

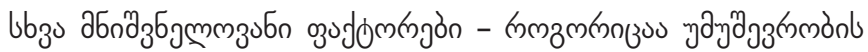

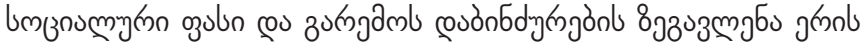

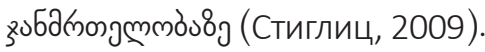

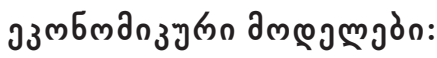

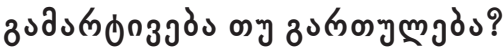

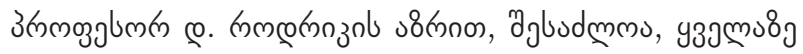

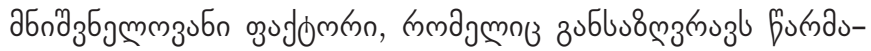

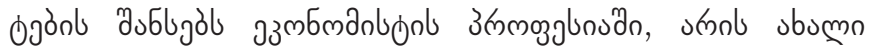

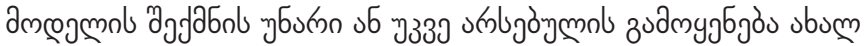

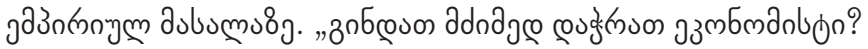

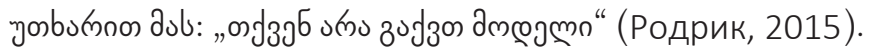

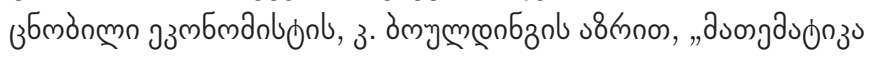

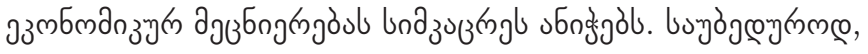

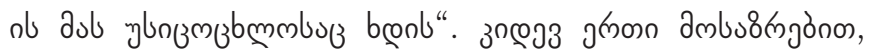

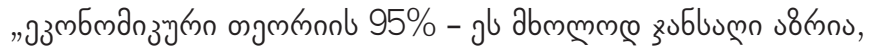

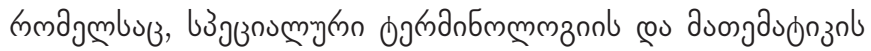

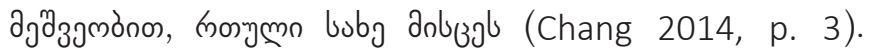

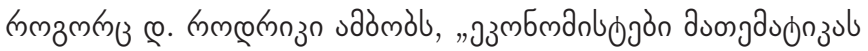

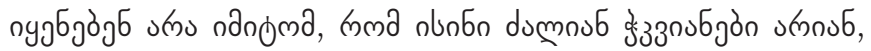

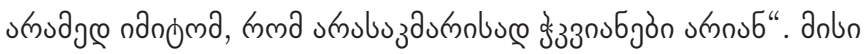

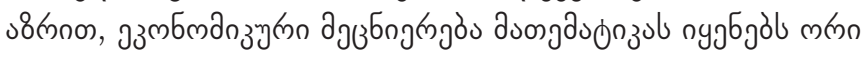

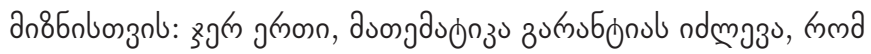

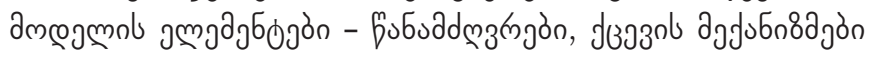

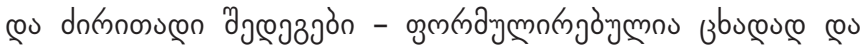

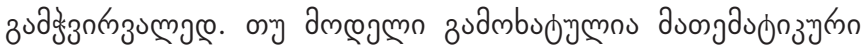

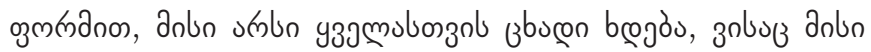

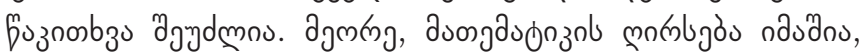

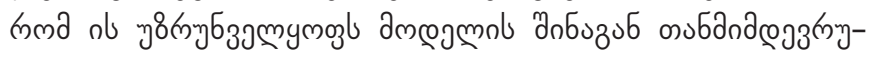

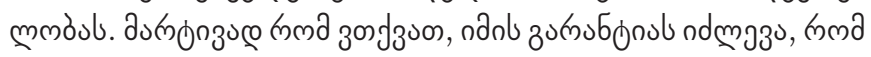

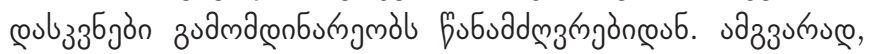

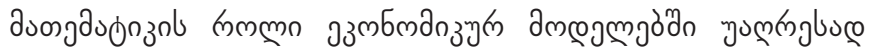

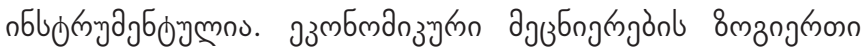
fumdmдuल

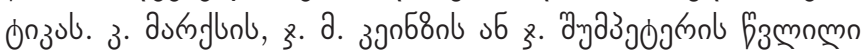

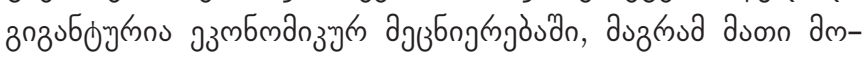

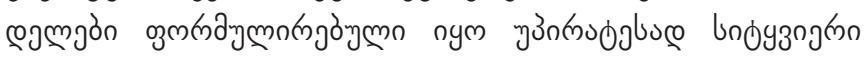

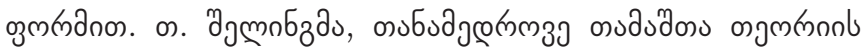

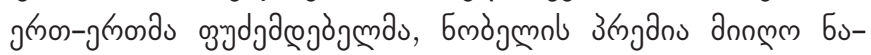

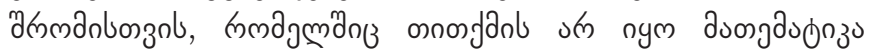

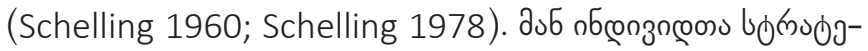

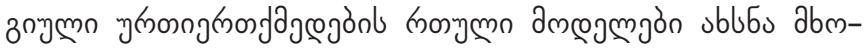

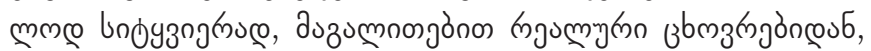




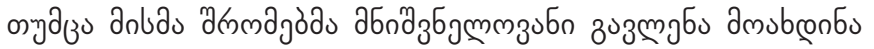

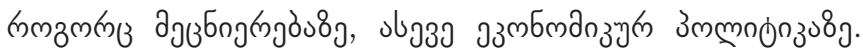

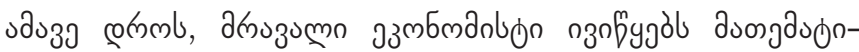

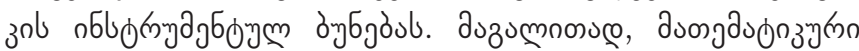

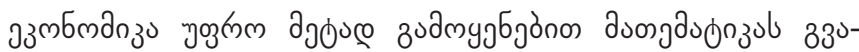

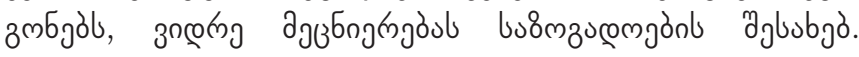

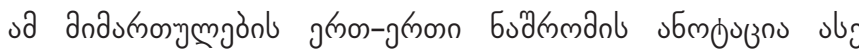
пf

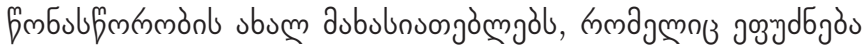

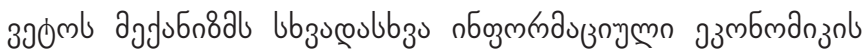

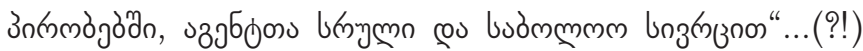

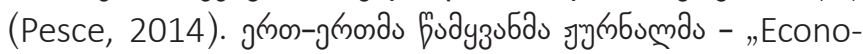

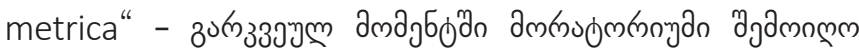

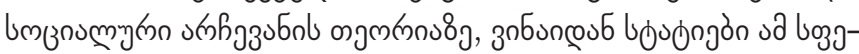

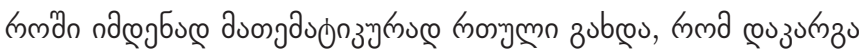

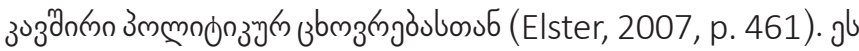

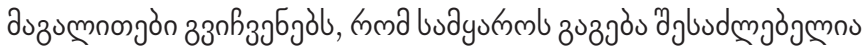

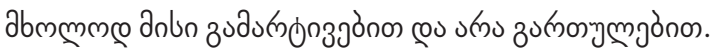

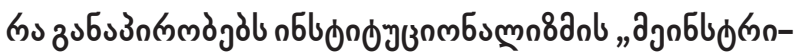

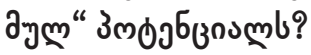

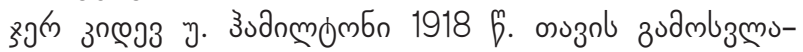

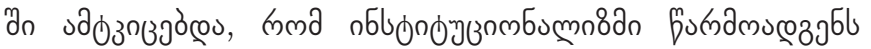

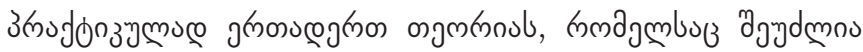

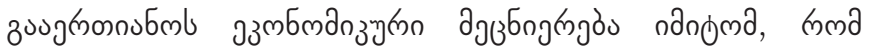
b 33 त

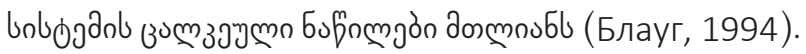

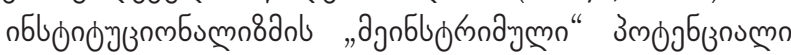

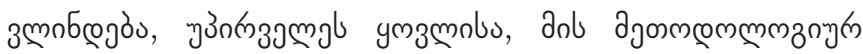

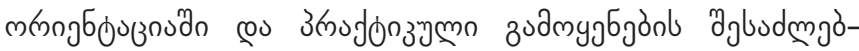

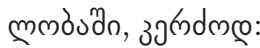

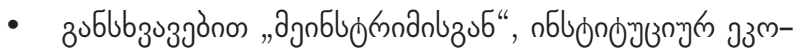

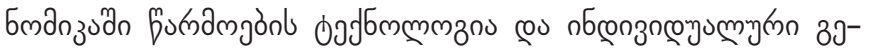

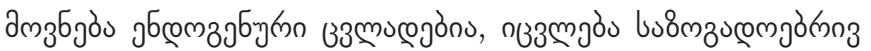

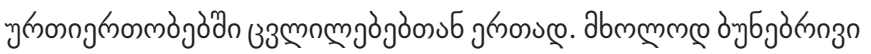

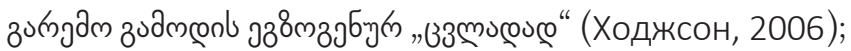

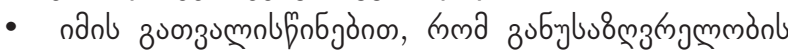
amb3mòs

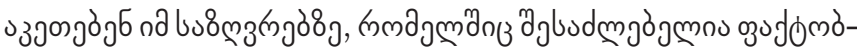

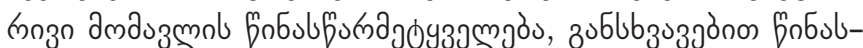

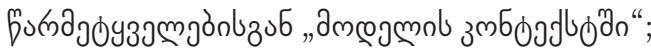

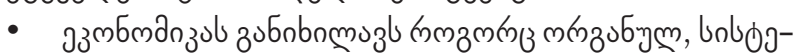

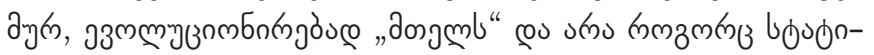

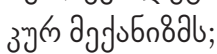

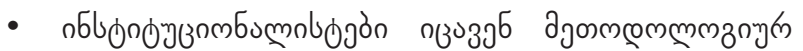

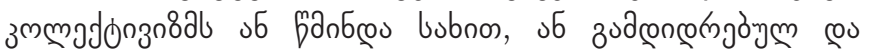

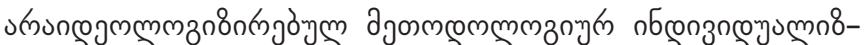

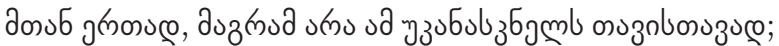

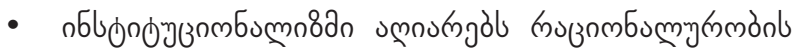

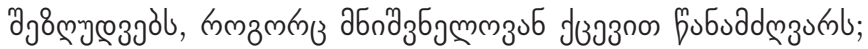

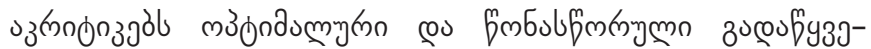

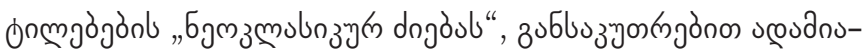

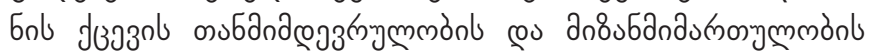
ozusmbugrnolnom;

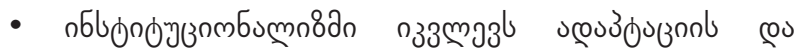

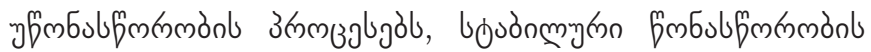

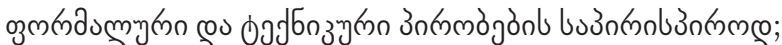

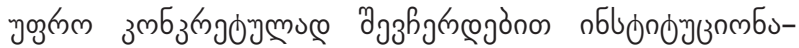

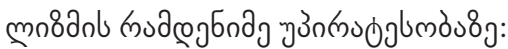

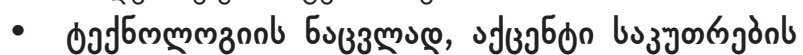

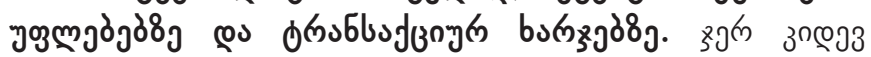

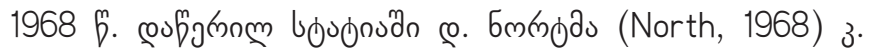

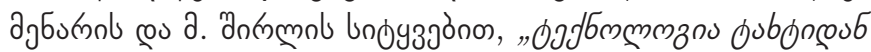

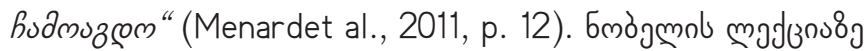

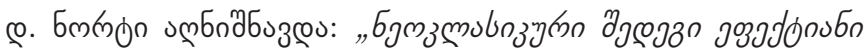

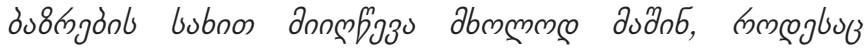

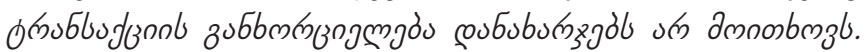

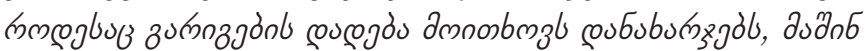

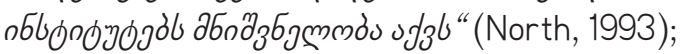

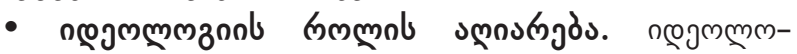

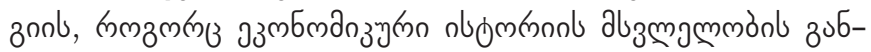

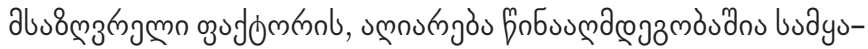

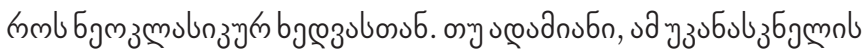

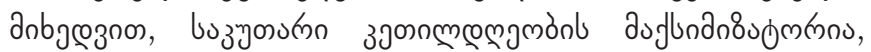

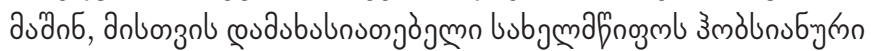

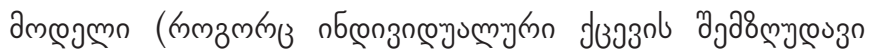

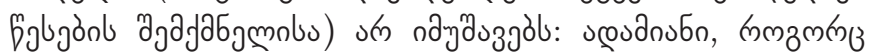

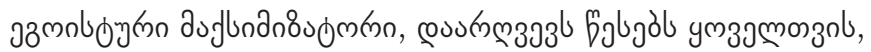

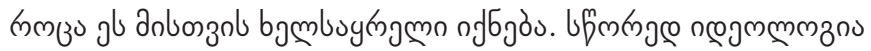

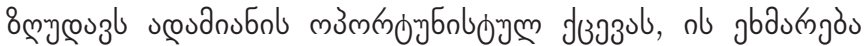

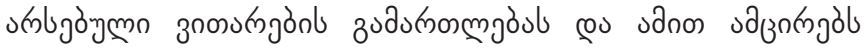

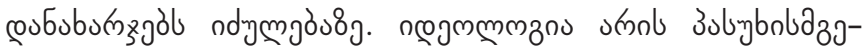

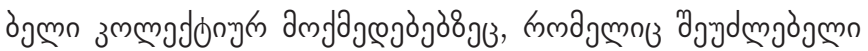

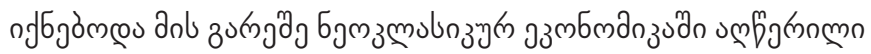

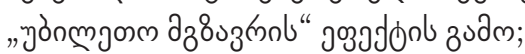

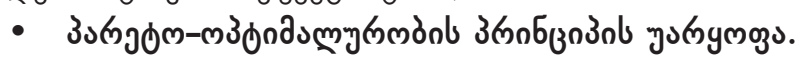

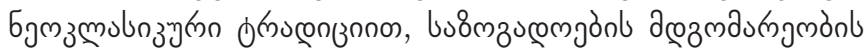

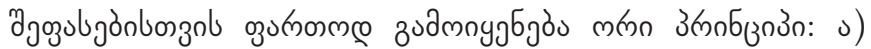

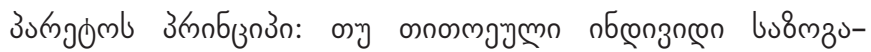

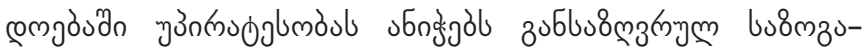

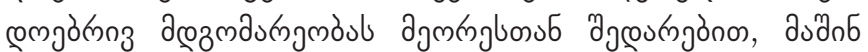

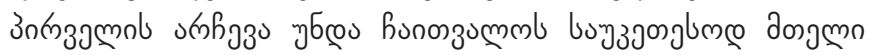

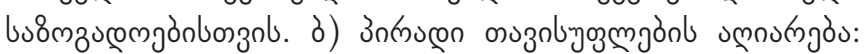

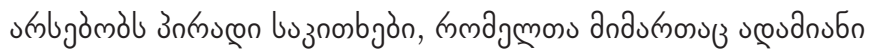

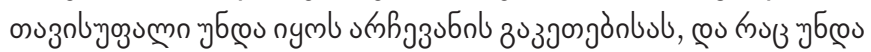

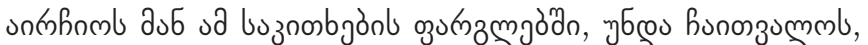

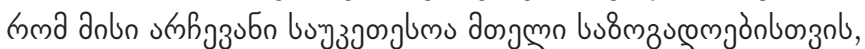

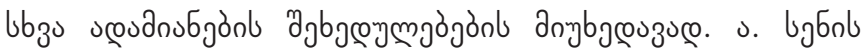




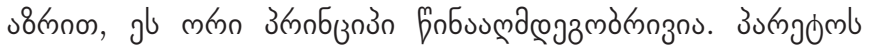

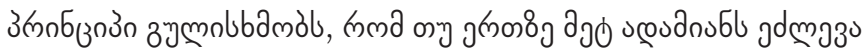

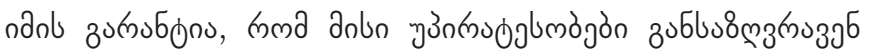

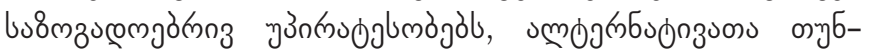

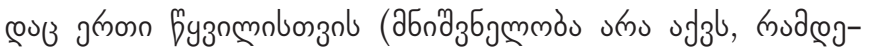

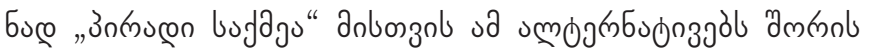

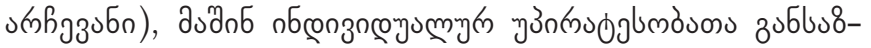

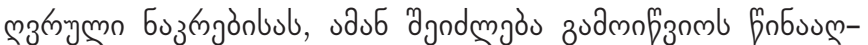

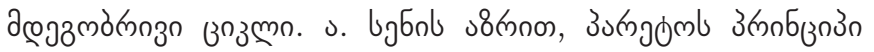

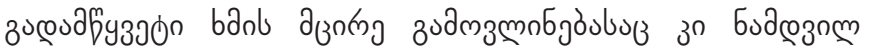

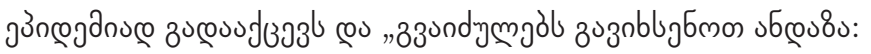

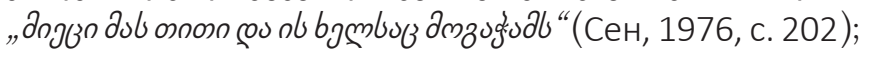

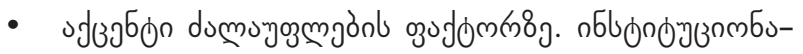

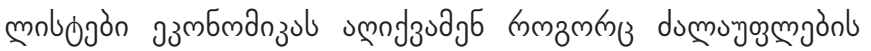

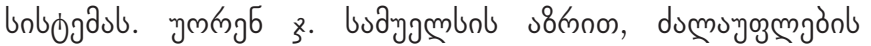

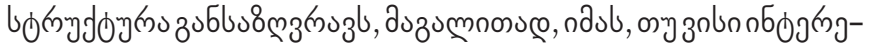

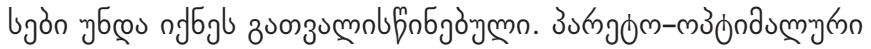

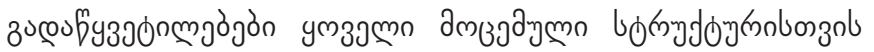

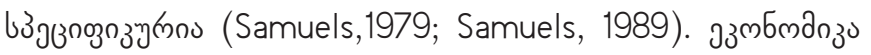

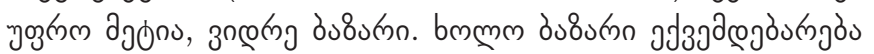

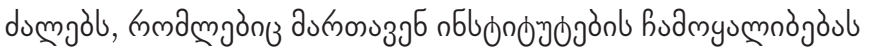

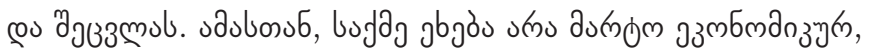

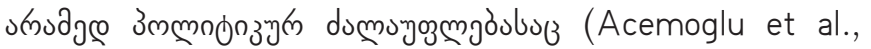

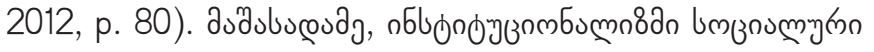

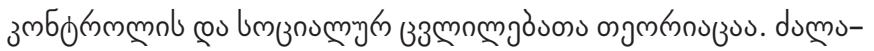

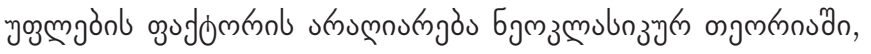

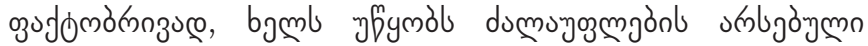
ь力ñydoynols maznondozoul.

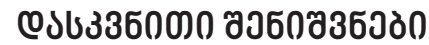

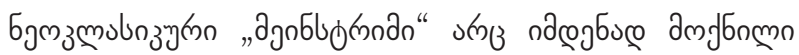

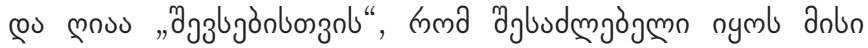

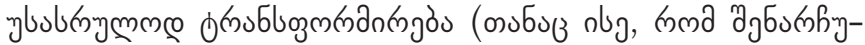
блбуym n nб

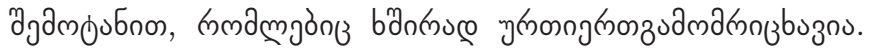

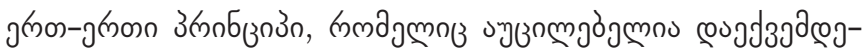

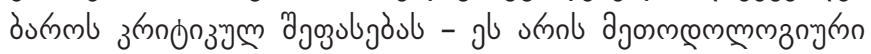

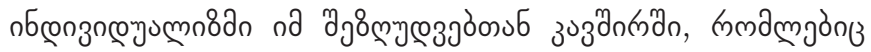

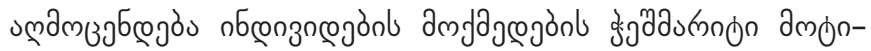

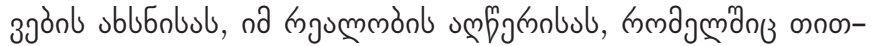

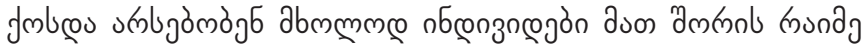

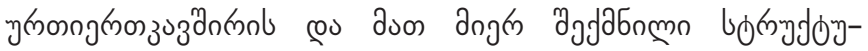

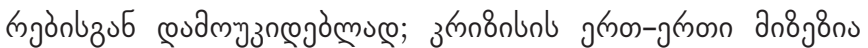

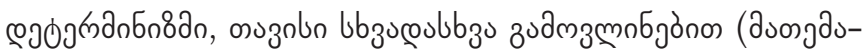

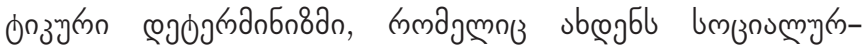

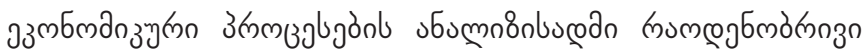

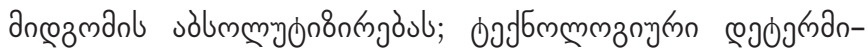

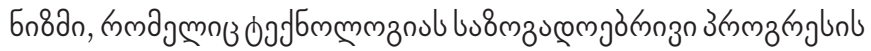

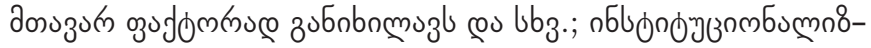

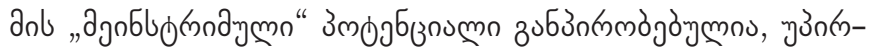

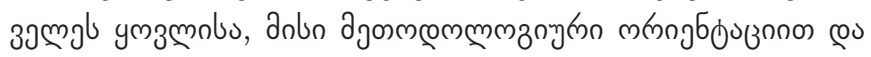

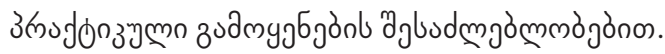




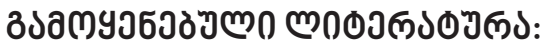

1. Acemoglu, D., Robinson, J.A. (2012). Why Nations Fail. The Origins of Power, Prosperity and Poverty. New York: Crown Business.

2. Chang, H.-J. (2014). Economics: The User Guide. London: Pelican Books.

3. Elster, J. (2007). Explaining Social Behavior: More Nuts and Bolts for the Social Sciences. Cambridge: Cambridge University Press.

4. Menard, C., Shirley, M. (2011). The Contribution of Douglass North to New Institutional Economics.https://halshs.archives-ouvertes.fr/file/index/docid/624297/filename/2011-Menard_Shirley_North_and_NIE--CUP.pdf).

5. North, D.C. (1981). Structure and Change in Economic History. NewYork-London.

6. North, D.C. (1968). Sources of Productivity Change in Ocean Shipping, 1600-1850. The Journal of Political Economy. Vol. 76, № 5.

7. North, D.C. (1993).Noble Prize Lecture. https://www.nobelprize.org/nobel_prizes/economic-sciences/laureates/1993/ north-lecture.html

8. Pesce, M.(2014). The Veto Mechanism in Atomic Differential Information Economies. Journal of Mathematical Economics, vol. 53, pp. 33-45.

9. Samuels, W., Tool, M. (eds). (1989). The Economy as a System of Power. New Brunswick, NJ: Transaction Books.

10. Schelling, T. C. (1960) The Strategy of Conflict, Cambridge, MA: Harvard University Press.

11. Schelling, T. C. (1978) Micromotives and Macrobehavior, New York: W. W. Norton.

12. Балацкий, Е. (2010). Кризис социальных наук в свете развития рынков. Общество и экономика. № 5.

13. Блауг, М. (1994). Экономическая мысль в ретроспективе. - М.: Дело,720 с.

14. Московский, А. (2012). Почему студенты Гарварда против лекций Г. Менкью. Экономист. № 1.

15. Норт, Д. (1997). Институты, институциональные изменения и функционирование экономики. М. «НАЧАЛА».

16.Родрик, Д. (2015). Экономика решает: сила и слабость «мрачной науки». Экономическая социология. Т. 16 . № 4.

17. Сен, А. К. (1976). Свобода, единогласие и права. http://www.seinstitute.ru/Files/veh4-1-6_Sen_p195-248.pdf

18. Стиглиц, Дж. (2009).нездоровый интерес к динамике ВВП привел к кризису. http://www.vedomosti.ru/finance/ news/2009/09/23/844148.

19. Фролов, Д. (2011). Теория кризисов после кризиса: технологии versus институты. Вопросы экономики. № 7.

20. Хелпман, Э. (2011).Загадка экономического роста. Пер. с англ. М.: Изд. Института Гайдара,- 240 с.

21. Ходжсон, Дж. (2006). Эволюционная и институциональная экономика как новый «мэйнстримз? Экономическийвестник Ростовского государственного университета. Т. 6. №2.

22. Ходжсон, Дж. (2003). Экономическая теория и институты. - М.: Дело. 464 с. 


\title{
THE PARADIGMATIC CRISIS AND THE "MAINSTREAM" POTENTIAL OF THE INSTITUTIONAL ECONOMICS
}

\author{
INGA BALARJISHVILI
}

https://doi.org/10.35945/gb.2018.06.015

Doctor of Economics, Associated Professor, Ivane Javakhishvili Tbilisi State University, Georgia

E-mail: Inga.balarjishvili@tsu.ge

\section{KEVWORDS: ECONOMIC SCIENCE'S CRISIS, "NEW-CLASSICALVVCTOR\#, INSTITUTIONAL ECONOMICS} maInStream" POTENTIAL, THE METHODOLOgICALPROBLEMS

\section{SUMMARY}

The article analyzes the manifestations and causes of the paradigmatic crisis of the modern economic "mainstream": the "monopolization" of economic theory and economic education by the neoclassical direction; Fragmentary nature of scientific research; The "fascination" of scientists with the problem of economic growth and ignoring other factors of social development. The emphasis is on the lack of interdisciplinary research, the "mathematization" of economic knowledge. It is noted that the role of mathematics in economic models is only instrumental, mathematically complex economic models make the world more incomprehensible than simplify, the connection with real life is lost.

Unlike the "mainstream», technology and individual taste in the institutional economy are endogenous variables. Institutionalists focus on the boundaries in which the actual future can be predicted, in contrast to the prediction in the «model context»; Institutionalists approach the economy as an organic and evolving "whole», and not as a static mechanism; Other advantages of the institutional economy are: the use of methodological collectivism instead of methodological individualism; Study of the processes of adaptation and disequilibrium, a criticism of the «neoclassical search» for optimal and equilibrium solutions; Instead of technology, focus on ownership and transaction costs; Recognition of ideology as the determining factor in economic history, which contradicts the neoclassical vision of the world; The refutation of the Pareto optimality principle; Emphasis on economic and political power.

The methodological orientation of the institutional economy and the possibility of practical application determine its potential for becoming a "new mainstream". 